Math. Model. Nat. Phenom.

Vol. 5, No. 7, 2010, pp. 4-10

DOI: $10.1051 / \mathrm{mmnp} / 20105701$

\title{
RBF Based Meshless Method for Large Scale Shallow Water Simulations: Experimental Validation
}

\author{
Y. Alhuri ${ }^{1}{ }^{*}$, A. Naji ${ }^{2}$, D. Ouazar ${ }^{3}$ and A. Taik ${ }^{1}$ \\ ${ }^{1}$ Dept of Mathematics, UFR-MASI, FST, Hassan II University, Mohammedia, Morocco \\ ${ }^{2}$ Dept of Mathematics, FST, Abdelmalek Essaadi University, Tanger, Morocco \\ ${ }^{3}$ Dept of Genie Civil, LASH, EMI, Mohammed V University, Rabat, Morocco
}

\begin{abstract}
D shallow water equations with depth-averaged $k-\varepsilon$ model is considered. A meshless method based on multiquadric radial basis functions is described. This methods is based on the collocation formulation and does not require the generation of a grid and any integral evaluation. The application of this method to a flow in horizontal channel, taken as an experimental device, is presented. The results of computations are compared with experimental data and are found to be satisfactory.
\end{abstract}

Key words: shallow water equations, depth-averaged $k-\varepsilon$ model, radial basis functions, collocation points.

AMS subject classification: 76F60,74G50

\section{Introduction}

Areas of modelling of turbulence in shallow water flows has not been treated so extensively as some other fluid dynamics. Some depth averaged turbulence models have been proposed for the 2D-Shallow Water Equation (2D-SWE) [7]. These models are derived from well known Reynoldsaveraged Navier-Stokes (RANS) turbulence models, including the effects of bed friction in the turbulence field. Special mention should be given to the depth averaged $k-\varepsilon$ model proposed by Rastogi and Rodi in 1978 [5], which was the first depth averaged two-equation eddy viscosity

\footnotetext{
${ }^{*}$ Corresponding author. E-mail: yasseralhuri@yahoo.com
} 
model, and it is still the most commonly used with the 2D-SWE when turbulent effects are included in the computation. J. Fe et al. [2] studied a finite volume turbulence model for the resolution of the two-dimensional shallow water equations with turbulence term, a comparison between $k-\varepsilon$ model and some experimental results is made. In this paper, a meshless method based on multiquadric radial basis functions approach is used to solve the 2D-SWE coupled to the two depth-averaged turbulence model. Obtained numerical results are compared with the experimental data given in [2] to validate the approach used.

\section{Governing equations, closure model}

The two-dimensional shallow water equations (2D-SWE) are obtained from the three-dimensional Navier-Sokes equations by means of an average in time, and integration in depth and some simplifying hypothesis [7], the most important of which is the hydrostatic pressure distribution, since the vertical length scale is much smaller than the horizontal one. The coefficient $\nu_{t}$ in the turbulent term of the 2D-SWE represent the eddy viscosity of a whole vertical column of fluid. To determine this viscosity different turbulence model can be used. One of them is the well-known $k-\varepsilon$ model, in which $k$ and $\varepsilon$ are, respectively, the turbulence kinetic energy and the dissipation rate per unit mass. The 2D-SWE are obtained from a depth integration, therefore it seems reasonable to use the two-dimensional depth-averaged version of the $k-\varepsilon$ model, in this model the eddy viscosity is calculated by $\nu_{t}=c_{\mu} \frac{k^{2}}{\varepsilon}$. Hence the two-dimensional shallow water equations with the depth-averaged turbulence model can be expressed as

$$
\frac{\partial \Phi}{\partial t}+\frac{\partial F}{\partial x}+\frac{\partial G}{\partial y}=M
$$

where $\Phi, F, G, E$ are vectors given as below

$$
\Phi=\left(\begin{array}{c}
h \\
h u=q_{u} \\
h v=q_{v} \\
h k=q_{k} \\
h \varepsilon=q_{\varepsilon}
\end{array}\right), F=\left(\begin{array}{c}
q_{u} \\
\frac{q_{u}^{2}}{h}+\frac{g h^{2}}{2} \\
\frac{q_{u} q_{v}}{h} \\
\frac{q_{u} q_{k}}{h} \\
\frac{q_{u} q_{\varepsilon}}{h}
\end{array}\right), G=\left(\begin{array}{c}
q_{v} \\
\frac{q_{u} q_{v}}{h} \\
\frac{q_{v}^{2}}{h}+\frac{g h^{2}}{2} \\
\frac{q_{v} q_{k}}{h} \\
\frac{q_{v} q_{\varepsilon}}{h}
\end{array}\right)
$$




$$
M=\left(\begin{array}{c}
0 \\
g h\left(S_{0 x}-S_{f x}\right)-\frac{\partial}{\partial x}\left(\nu_{t} \frac{\partial q_{u}}{\partial x}\right)-\frac{\partial}{\partial y}\left(\nu_{t} \frac{\partial q_{u}}{\partial y}\right) \\
g h\left(S_{0 y}-S_{f y}\right)-\frac{\partial}{\partial x}\left(\nu_{t} \frac{\partial q_{v}}{\partial x}\right)-\frac{\partial}{\partial y}\left(\nu_{t} \frac{\partial q_{v}}{\partial y}\right) \\
-\frac{\partial}{\partial x}\left(\frac{\nu_{t}}{\sigma_{k}} \frac{\partial q_{k}}{\partial x}\right)-\frac{\partial}{\partial y}\left(\frac{\nu_{t}}{\sigma_{k}} \frac{\partial q_{k}}{\partial y}\right)-S_{k}(\Phi) \\
-\frac{\partial}{\partial x}\left(\frac{\nu_{t}}{\sigma_{\varepsilon}} \frac{\partial q_{\varepsilon}}{\partial x}\right)-\frac{\partial}{\partial y}\left(\frac{\nu_{t}}{\sigma_{\varepsilon}} \frac{\partial q_{\varepsilon}}{\partial y}\right)-S_{\varepsilon}(\Phi)
\end{array}\right)
$$

Here $x$ and $y$ are the horizontal coordinates, $t$ is the time, $h=h(x, y, t)$ is the instantaneous total depth of water, $u=u(x, y, t)$ is the depth-averaged velocity in the $x$ direction, $v=v(x, y, t)$ is the depth-averaged velocity in the $y$ direction. $S_{0 x}$ and $S_{0 y}$ are the bottom bed slopes defined by : $S_{0 x}=-\frac{\partial z_{f}}{\partial x}$ and $S_{0 y}=-\frac{\partial z_{f}}{\partial y}, z_{f}$ being the bed elevation. $S_{f x}$ and $S_{f y}$ correspond to the bottom friction slopes, which are approximated by the Manning formula $S_{f x}=\frac{n^{2} u \sqrt{u^{2}+v^{2}}}{h^{4 / 3}}, S_{f y}=$ $\frac{n^{2} v \sqrt{u^{2}+v^{2}}}{h^{4 / 3}}$, where $n$ is the Manning roughness coefficient, $g$ is the gravitational acceleration. The Coriolis and the stresses terms have been neglected. The reason is that the Coriolis term has little significance when applied to small domains and the wind term influence is not relevant in some situations such as indoor laboratory channels. $k$ and $\varepsilon$ are respectively the average of the kinetic turbulent energy and the average of its dissipation rate, $\nu_{t}$ is a turbulent eddy viscosity, computed from the $k-\varepsilon$ turbulence model, and

$$
\begin{gathered}
P_{k}=\frac{1}{\sqrt{c_{f}}} u^{* 3}, \quad u^{*}=\sqrt{\frac{\tau_{w}}{\rho_{\omega}}}, \quad S_{\varepsilon}=c_{1 \varepsilon} \frac{\varepsilon}{k} P_{h}+P_{\varepsilon v}-c_{2 \varepsilon} \frac{h \varepsilon^{2}}{k}, \quad P_{\varepsilon v}=c_{\varepsilon} \frac{u^{* 4}}{h}, \\
S_{k}=P_{h}+P_{k}-h \varepsilon, \quad P_{h}=\frac{\nu_{t}}{h}\left[2\left(\frac{\partial q_{u}}{\partial x}\right)^{2}+2\left(\frac{\partial q_{v}}{\partial y}\right)+\left(\frac{\partial q_{u}}{\partial y}+\frac{\partial q_{v}}{\partial x}\right)^{2}\right],
\end{gathered}
$$

where $P_{h}$ is the production of $k$ due to interactions of turbulent stresses with horizontal meanvelocity gradients. $P_{k}$ and $P_{\varepsilon v}$ are the productions of $k$ and $\varepsilon$ due to vertical velocity gradients and are related to the friction velocity $u^{*}$, which calculated from the so-called law of the wall and $\tau_{w}$ is the wall stress, $\rho_{\omega}$ is the density of water. $c_{\mu}=0.09, c_{1 \varepsilon}=1.44, c_{2 \varepsilon}=1.92, \sigma_{k}=1.0, \sigma_{\varepsilon}=1.3$, $c_{f}=5.19$ and $\kappa=0.41$ are an empirical constants [2].

\section{Radial basis functions approach}

The theory of radial basis functions approximation have been discussed comprehensively in [1]. The principal idea of the radial basis interpolation is to interpolate a finite series of an unknown 
function $f(\mathbf{X})$ at $N$ distinct points $\mathbf{X}_{j}$ on $\Omega$ by the following expansion

$$
f(\mathbf{X}) \simeq \sum_{j=1}^{N} \alpha_{j} \varphi\left(\left\|\mathbf{X}-\mathbf{X}_{j}\right\|\right)
$$

where $\varphi\left(\left\|\mathbf{X}-\mathbf{X}_{j}\right\|\right)$ is the radial basis function, $X_{j} \in \mathbb{R}^{2}, j=1,2, \ldots, N,\left\|\mathbf{X}-\mathbf{X}_{j}\right\|=r_{j}$ is the Euclidean distance and $\mathbf{X}=\left(x_{i}, y_{i}\right), \mathbf{X}_{j}=\left(x_{j}, y_{j}\right)$, and $\alpha_{j}$ 's are the unknown coefficients to be calculated at each time step. The most popular RBFs are multiquadric $(M Q R B F)[4], \varphi\left(r_{j}\right)=$ $\left(r_{j}^{2}+c^{2}\right)^{1 / 2}=\sqrt{\left(x_{i}-x_{j}\right)^{2}+\left(y_{i}-y_{j}\right)^{2}+c^{2}}$ are infinitely smooth functions, $c \neq 0$ is the shape parameter controlling the fitting of a smooth surface to the data [3]. To solve the two-dimensional time-dependent differential equations given by equation (2.1), it is integrated in time using forward difference scheme given as

$$
\Phi_{i}^{n+1}=\Phi_{i}^{n}-\Delta t\left(\frac{\partial G_{i}^{n}}{\partial x}+\frac{\partial F_{i}^{n}}{\partial y}+M_{i}^{n}\right),
$$

where $\Delta t$ is the time step, $\Phi_{i}^{n}$ is the numerical solution vector at points $\left(x_{i}, y_{i}\right)$ in time $n \Delta t$. The values of the interpolant $\Phi^{n}$ are given by the following $M Q$ radial basis function

$$
\Phi^{n}\left(x_{i}, y_{i}, t\right)=\sum_{j=1}^{N} \alpha_{j}^{n}(t) \sqrt{r_{j}^{2}+c^{2}},
$$

which are collocating with a set of data $\left(x_{i}, y_{i}\right)_{i=1}^{N}$ over the domain $\Omega \subset \mathbb{R}^{2}$. This equation forms a system of $N$ linear equations in $\mathrm{N}$ unknowns which can be expressed in matrix form

$$
\vec{\Phi}=A \vec{\alpha}
$$

where $A=\left[\varphi_{j}\left(x_{i}, y_{i}\right)\right]$ is a $N \times N$ coefficient matrix, $\vec{\alpha}=\left[\alpha_{j}^{n}\right]$ and $\vec{\Phi}=\left[\Phi_{j}^{n}\right]$ are $N \times 1$ matrices. The unknown coefficients vector $\left[\alpha_{j}^{n}\right]$ can be determined using Gaussian elimination or GMRS method. It follows that the numerical values for the corresponding spatial derivatives of $\Phi^{n}\left(x_{i}, y_{i}\right)$ can be calculate using equation (3.3). The solution of the variables $\left(H, q_{u}, q_{v}, q_{k}, q_{\varepsilon}\right)$ is solved by substituting the partial derivatives into equation (3.2) with given boundary conditions. Finally, the time step $\Delta t=t_{n+1}-t_{n}$ is estimated by the Courant-Friedrichs-Lewy condition [6]

$$
\Delta t \leq C \frac{d_{\min }}{\max (u \pm \sqrt{g h}, v \pm \sqrt{g h})}
$$

where $d_{\min }$ is the minimum distance between any two adjacent collocation points and $C$ is the Courant number set to be less than unity to ensure the stability of the scheme.

Before presenting the numerical results let us discuss the boundary conditions. At the inlet and outlet the usual conditions for the hydrodynamic equations are applied (see [2] and references therein). If the flow is subcritical, the discharge at the inlet and the depth at the outlet must be imposed. For the $k-\varepsilon$ process, the most adequate inlet condition would be of Dirichlet type, measuring $k$ experimentally and obtaining $\varepsilon$ from other turbulent quantities, conditions very common in the literature [2] have been adopted for the wall and outlet sections, they are of Dirichlet-type at the walls and of Neumann-type at the outlet, for more details see [2]. 


\section{Numerical results and experiment validation}

The experimental device consisted of a horizontal channel made of glass with a sudden expansion. The dimensions of this device are indicates in Figure 1. The experimental data were obtained at the Hydraulics Laboratory of Civil Engineering School of A Coruna (Spain) [2].

As shown in Figure 2 we generate $N=427$ collocation points, where 338 collocation points are in the interior, 19 collocation points are on the water boundary (inlet and outlet) and 70 are on the land boundary. Multiquadric radial basis functions approach do not require triangulation mesh of the domain. The model was applied with turbulent term $k-\varepsilon$ phase. The results obtained by

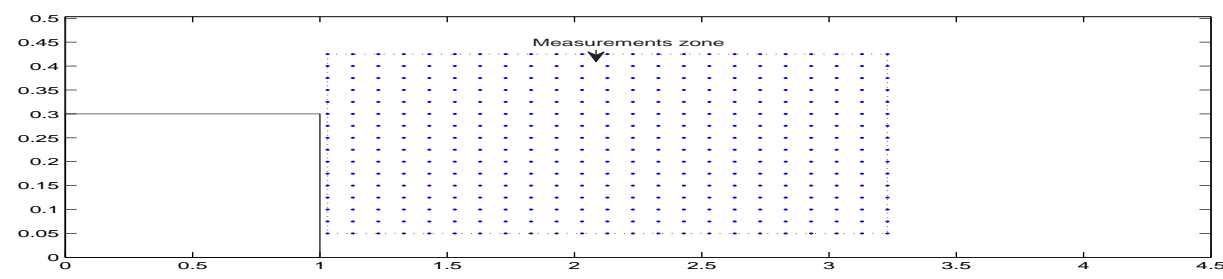

Figure 1: General view of the experimental device with measurement zone.

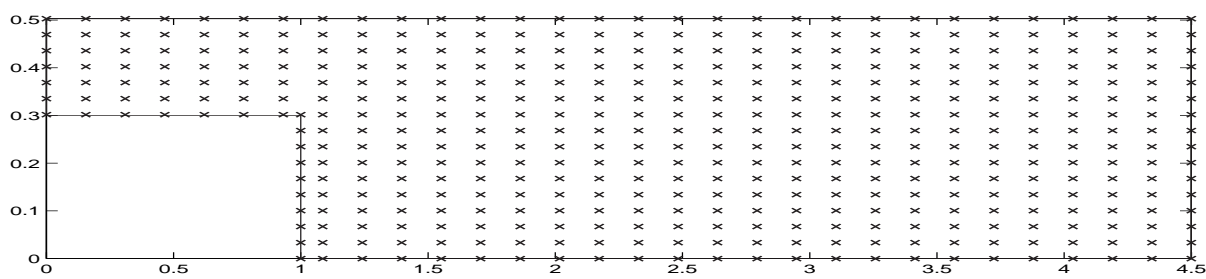

Figure 2: RBF collocation points.

$M Q R B F$ method and experimental data of the water velocity $u$ and turbulent kinetic energy $k$ at sections $x_{1}=1.53 \mathrm{~m}, x_{2}=2.03 \mathrm{~m}, x_{3}=2.53 \mathrm{~m}$ are presented in Figures 3, 6. Figures 4, 5 show respectively the experimental data of the velocity field and streamlines and the obtained numerical results using the two-dimensional shallow water equations with depth-averaged turbulence model. The velocity vectors and streamlines obtained are very close to the experimental measurements.

\section{Conclusion}

The two-dimensional shallow water equations with depth-averaged turbulence $k-\varepsilon$ model has been described. A global multiquadric radial basis functions method was applied to solve the resulting model. Since it is a meshless method its implementation is simple contrary to the others 

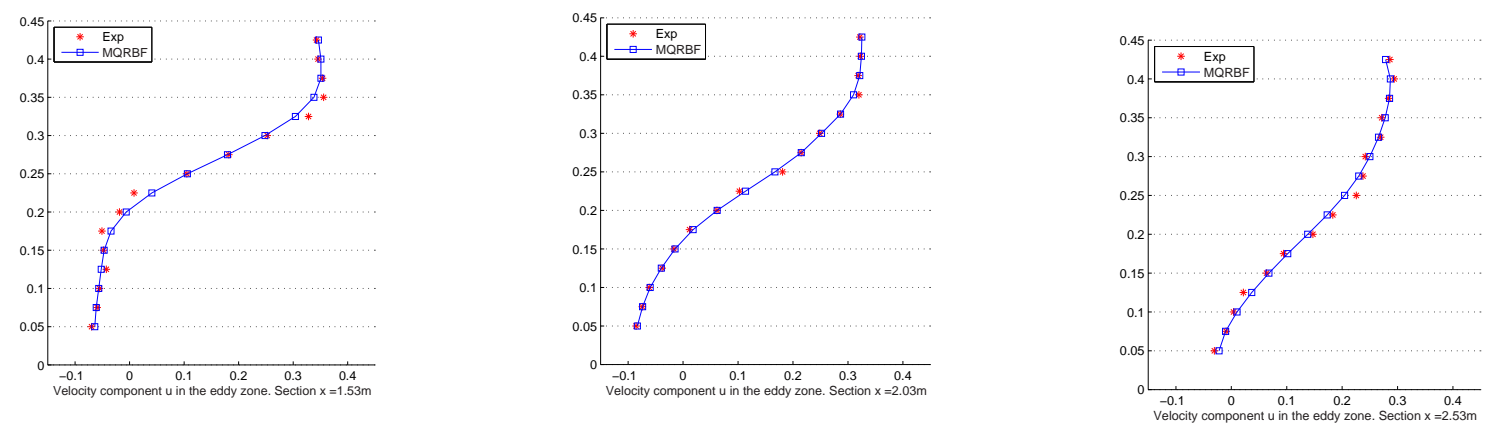

Figure 3: Experimental and numerical profiles of $u$ for sections $x=1.53 \mathrm{~m}, 2.03 \mathrm{~m}, 2.53 \mathrm{~m}$.

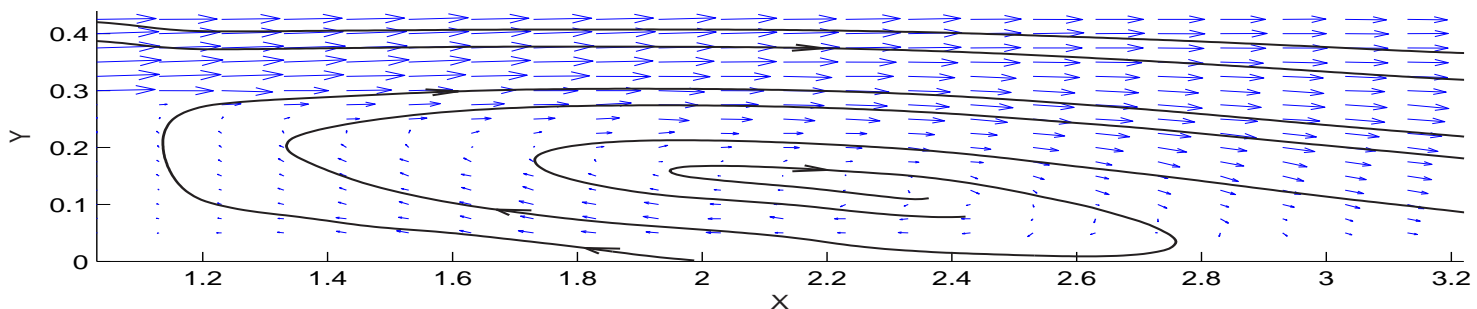

Figure 4: Experimental measures. Streamlines and velocity profiles.

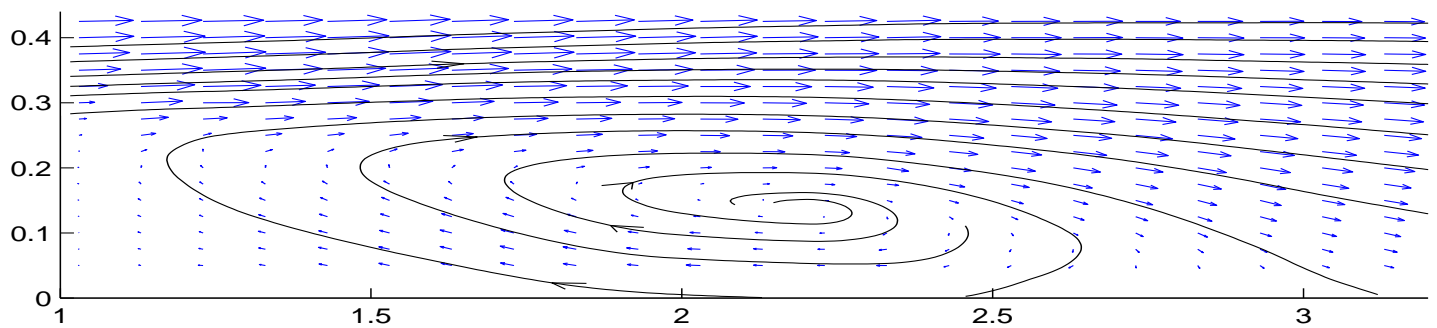

Figure 5: 2D-SWE with $k-\varepsilon$ model. Streamlines and velocity profiles.
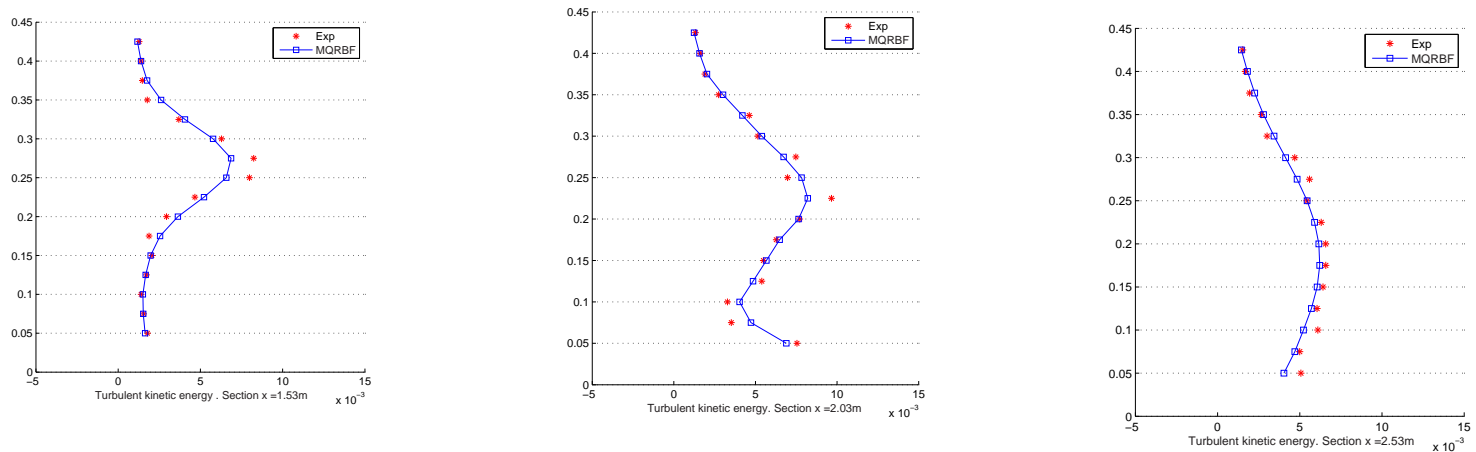

Figure 6: Experimental and numerical profiles of $k$ for sections $x=1.53 m, 2.03 m, 2.53 m$. 
methods like finite volumes or finite elements. The numerical results seems in good agrement with the given experimental data.

The results, obtained with the proposed global radial basis functions, can be considered very satisfactory. Some improvements could be expected if compactly supported radial basis functions [1] were used, although the computational cost should also be lower since the resulting matrix is sparse.

\section{Acknowledgements}

The authors would like to thank Professor J. Fe (University of A Coruna, Spain) for his kind help in giving us the experimental data used to validate our approach in this paper.

\section{References}

[1] M. Buhamman. Radial basis functions: theory and implementations. Cambridge University Press, 2003.

[2] J. Fe, F. Navarrina, J. Puertas, P. Vellando, D. Ruiz. Experimental validation of two depthaveraged turbulence models. Int. J. Numer. Meth. Fluids, 60 (2009), 177-202.

[3] E.J. Kansa, R. E. Carlson. Improved accuracy of multiquadric interpolation using variable shape parameters. Comput. Math. Applic., 24 (1992), No.12, 99-120.

[4] E.J. Kansa. Multiquadrics a scattered data approximation scheme with application to computational fluid dynamics. Part I, Comput. Math. Applic., 19 (1990), 127-145.

[5] A. Rastogi, W. Rodi. Predictions of heat and mass transfer in open channels. Journal of the Hydraulics Division, proceedings of the American Society of Civil Engineers, 104 (HY3) (1978), 397-418.

[6] S.M. Wong. Meshless methods for large-scale computations by radial basis functions. Ph.D. Thesis, City Univ. Hong Kong, China, 2001.

[7] C.B. Vreudgenhil. Numerical methods for shallow-water flow. Kluwer Academic Publishers: Dordrecht, The Netherlands, 1994. 